\title{
Teoria das Restrições: Um Estudo Bibliométrico da Produção Científica Apresentada no Congresso Brasileiro de Custos
} (1994-2008)

\begin{abstract}
Claudia Cruz
Mestrado em Ciências Contábeis pela Universidade Federal do Rio de Janeiro - UFRJ Av. Pasteur, 250. Sala 249. Urca (UFRJ, FACC). Rio de Janeiro/RJ. CEP: 22290-240

E-mail: claudiacruzba@gmail.com

Alessandra Marques

Mestrado em Ciências Contábeis pela Universidade Federal do Rio de Janeiro - UFRJ Av. Pasteur, 250. Sala 249. Urca (UFRJ, FACC). Rio de Janeiro/RJ. CEP: 22290-240

E-mail: alessandralmarques@hotmail.com

Roberval Silva

Mestrado em andamento em Ciências Contábeis pela Universidade Federal do Rio de Janeiro - UFRJ

Av. Pasteur, 250. Sala 249. Urca (UFRJ, FACC). Rio de Janeiro/RJ. CEP: 22290-240 E-mail: roberval.rubens@uol.com.br

Doutorado em Engenharia de Produção pela Universidade Federal do Rio de Janeiro UFRJ

Professor da Universidade Federal do Rio de Janeiro - UFRJ Av. Pasteur, 250. Sala 249. Urca (UFRJ, FACC). Rio de Janeiro/RJ. CEP: 22290-240 E-mail:scogan@uol.com.br
\end{abstract}

\section{RESUMO}

O objetivo principal da pesquisa em questão é analisar os trabalhos científicos com abordagem no tema "Teoria das Restrições" (TOC), apresentados no Congresso Brasileiro de Custos (CBC) de 1994 a 2008, por meio de um estudo bibliométrico. A TOC foi desenvolvida por Eliyahu Goldratt a partir dos anos 1970 para contribuir na resolução dos problemas de gestão e de controle de custos em ambientes fabris e na melhoria contínua no desempenho esperado de qualquer organização. Por meio de uma pesquisa documental nos 47 artigos sobre o tema apresentados em todas as edições do CBC, os resultados revelam que: a maioria dos trabalhos tinha dois autores; dois terços dos autores são do sexo masculino; as regiões Sudeste e Sul concentram 92,6\% dos trabalhos; a Universidade Federal do Rio de Janeiro foi a instituição mais recorrente e os autores mais prolíficos foram: Samuel Cogan, Joanília Neide de Sales Cia e Anselmo Rocha Neto; os enfoques mais abordados buscaram comparar ou integrar as premissas da TOC com outros métodos de controle e gestão de custos; as estratégias de pesquisa mais adotadas foram pesquisa bibliográfica e estudo de caso; a maioria os trabalhos teve finalidade descritiva ou exclusivamente teórica; a média de referências por artigo foi 15,7 e a categoria referencial mais citada foi livros. A 
concentração das referências bibliográficas em livros, as limitadas consultas a periódicos, teses e dissertações e anais de congressos denotam um posicionamento conservador e convencional dos autores e um estágio incipiente das pesquisas com abordagem no tema Teoria das Restrições.

Palavras-chave: Teoria das Restrições. Bibliometria. Congresso Brasileiro de Custos.

\section{Theory of Constraints: A Bibliometric Study of Scientific Production Presented at the Brazilian Congress of Costs (1994-2008)}

\section{ABSTRACT}

The main objective of this research is to analyze the scientific approach with the theme "Theory of Constraints", presented at Congresso Brasileiro de Custos (CBC) from 1994 to 2008, through a bibliometric research. The TOC was developed by Eliyahu Goldratt from the 70's to help in solving the problems of management and cost control in manufacturing environments and continuous improvement in performance expected from any organization. Through documentary research in 47 papers on the subject presented in all editions of $\mathrm{CBC}$, the results show that: almost all the papers have two authors, two-thirds of them are male, the Southeast and South of Brazil contain 92, 6\% of the papers, the Federal University of Rio de Janeiro(UFRJ) was the institution with most recurrent and the most prolific authors were: Samuel Cogan, Joanília Neide de Sales Cia and Anselmo Rocha Neto, the most frequently discussed approaches attempting to compare or integrate the premises of TOC with other methods of control and cost management, the research strategies adopted were more literature and case study, most of the papers were intended exclusively descriptive or theoretical, the average number of citations per article was 15.7 and the reference category most cited were books. The concentration of references in books, the limited consultations in journals, theses and dissertations and conference proceedings show a conservative and conventional positioning of the authors and an incipient stage of research, addressing the theme Theory of Constraints.

Key Words: Theory of Constraints. Bibliometric Research. Brazilian Congress of Costs.

\section{INTRODUÇÃO}

Dentre tantos métodos e processos de gestão e de controle de custos desenvolvidos com o objetivo de otimizar a utilização dos recursos durante o processo produtivo e maximizar os resultados positivos das organizações, encontra-se a Teoria das Restrições (TOC), desenvolvida a partir da década de 1970 pelo físico israelense 
Eliyahu Goldratt. Com o objetivo de minimizar as dificuldades encontradas no processo de fabricação, que de antemão foram por ele identificadas, a Teoria das Restrições foi desenvolvida para contribuir no equacionamento desses problemas em ambientes semelhantes e na promoção da melhoria contínua no desempenho esperado de qualquer organização.

Como uma ferramenta de sucesso utilizada por algumas empresas, após a edição do livro "A Meta" em 1984, em que apresenta os conceitos básicos da Teoria das Restrições, Goldratt publicou seu segundo livro "A corrida pela vantagem competitiva" (1985), apresentando uma contribuição técnica para promover melhorias nas empresas por meio de um processo de aprimoramento contínuo.

Essa importante contribuição à Contabilidade Gerencial e de Custos baseia-se na premissa de que devido à impossibilidade de se rastrear os custos fixos com precisão, as empresas deveriam considerar o lucro total e não o custo de cada produto. Para que esse conceito seja praticado, deve haver uma mudança de paradigma nos administradores, na medida em que o "mundo dos custos" é substituído pelo "mundo dos ganhos".

Ao considerar a relevância do tema e a expansão das pesquisas na área de Administração e Contabilidade e, seguindo a tendência de pesquisas que têm como objeto de estudo a produção científica, a presente pesquisa tem como propósito analisar os trabalhos científicos com abordagem do tema "Teoria das Restrições", apresentados no Congresso Brasileiro de Custos (CBC) de 1994 a 2008, por meio do estudo bibliométrico. Para consecução desse objetivo foram analisadas algumas variáveis específicas: perfil dos autores (sexo, vinculação institucional e estado de origem); quantidades de autores em cada artigo; enfoques temáticos empregados; procedimentos metodológicos adotados; e categorias referenciais citadas nos artigos.

Assim, esse trabalho apresenta, inicialmente, um referencial teórico acerca da origem e desenvolvimento da Teoria das Restrições e um resumo de suas principais características e conceitos. No item dois, são apresentados conceitos e metodologias sobre a abordagem bibliométrica. A seguir são descritos a metodologia adotada, bem como os resultados da pesquisa e as conclusões, seguidos das referências. 


\section{REFERENCIAL TEÓRICO}

\subsection{Teoria das Restrições}

A Teoria das Restrições (do original inglês Theory of Constraints - TOC) foi desenvolvida pelo físico israelense Eliyahu Goldratt na década de 1970, a partir da sua experiência na concepção de um projeto de logística de produção, em que desenvolveu um software de programação de produção chamado OPT (Optimized Production Technology). No entanto, foi a partir da publicação do livro The Goal (no Brasil conhecido como A Meta) em 1984, em parceria com Jeff Cox que suas idéias foram compiladas e amplamente divulgadas (COGAN, 2007; ROGERS e REIS, 2005) e o arcabouço das idéias da Teoria das Restrições ganhou divulgação mundialmente (GUERREIRO, 1996).

A motivação de Goldratt para desenvolver a TOC teve origem em sua percepção de que os métodos e princípios destinados às pesquisas científicas poderiam também ser aplicados às áreas de negócios e, principalmente, à manufatura (GOLDRATT; COX, 1992). Como estudioso da Física, Goldratt analisou o ambiente da fábrica numa perspectiva diferente e passou a aplicar conceitos da Física, a exemplo das relações de 'Causa e Efeito', para compreender a lógica de funcionamento de setor de produção. Suas análises permitiram uma compreensão simplificada de questões antes sem solução, permitindo a resolução de antigos problemas de maneira simples e eficiente. Essa experiência na busca de compreender os problemas de programação da fábrica constitui a origem do que hoje se conhece como Teoria das Restrições.

Conforme definição de Cogan (2007, p. 293), a Teoria das Restrições constitui uma filosofia de gerenciamento holístico que enuncia métodos para maximizar o resultado operacional diante de gargalos (restrições) nas operações. Para Silva et al. (2007), a TOC pode ser considerada como uma filosofia de operação, cujo foco é a gestão de atividades que restringem o desempenho da organização, e visa um processo de melhoria contínua da mesma. De forma mais ampla, a TOC pode ser definida também como uma filosofia global de gerenciamento empresarial, cujo objetivo maior é promover a contínua melhoria do desempenho esperado de qualquer 
organização que tenha uma meta bem definida, por meio do foco nas ações gerenciais nos elementos que restringem o alcance da meta (LINTER, 2002).

De forma semelhante, MacArthur (1993) menciona que a TOC é considerada uma filosofia de ambientes fabris, cujo objetivo é maximizar lucros por meio de uma gestão adequada dos gargalos ou restrições, que tem ênfase no curto prazo com implicações de longo prazo. Por sua vez, Giuntini et al. (2002) vêem a TOC como uma inovação dentro do campo da Contabilidade Gerencial ao significar uma nova forma de 'ver e pensar' o gerenciamento de uma empresa, cujo objetivo é contribuir com novos princípios e práticas para o desenvolvimento da Contabilidade Gerencial. Em outro trabalho, Cogan (1999) acrescenta que a Teoria das Restrições desenvolvida por Goldratt aparenta ser um refinamento do custeio direto associado com técnicas de programação linear.

Embora, a origem da TOC tenha se dado a partir da análise das dificuldades encontradas no setor de produção de uma fábrica, Goldratt não ficou restrito a essa realidade, mas vislumbrou que poderia contribuir para o equacionamento de problemas semelhantes encontrados em outros ambientes e contextos. A partir de então, desenvolveu conceitos que atribuíssem uma estrutura mais completa às idéias iniciadas na fábrica para dar um corpo à sua teoria.

A TOC tem como premissa básica o gerenciamento de limitações, restrições ou gargalos e considera que toda a organização (empresarial ou não) possui uma meta principal que enfrenta restrições internas e/ou externas para ser alcançada. Em se tratando das organizações empresariais, "a meta é ganhar dinheiro no presente e no futuro" e a maneira de alcançá-la é incrementar o ganho da empresa. Porém, o ganho está limitado por gargalos e o caminho para o alcance da meta está em administrar os gargalos (restrições do sistema). Assim, gargalo ou restrição, conceito seminal da TOC, é todo recurso cuja capacidade é igual ou menor do que a demanda existente (COGAN, 2007).

A partir da definição da meta da organização e da identificação das restrições existentes, a TOC oferece diversos conceitos e técnicas para melhorar o desempenho da organização com vistas a incrementar o seu ganho e alcançar a meta. 
Teoria das Restrições: Um Estudo Bibliométrico da Produção Científica Apresentada no
Congresso Brasileiro de Custos (1994-2008)
Claudia Cruz, Alessandra Marques, Roberval Silva, Samuel Cogan

O Quadro 1 apresenta um resumo dos principais conceitos relacionados com a Teoria das Restrições e que são mais recorrentemente adotados e discutidos na literatura específica.

\begin{tabular}{|c|c|}
\hline Parâmetros/Conceitos & Características da Teoria das Restrições \\
\hline Fundador & Físico israelense Eliyahu Goldratt \\
\hline Período de surgimento & Década de 70 (Século XX) \\
\hline $\begin{array}{l}\text { Principais obras } \\
\text { relacionadas }\end{array}$ & $\begin{array}{c}\text { "A Meta" (1984); “A corrida pela vantagem competitiva" (1985); "A } \\
\text { síndrome do palheiro: garimpando informação num oceano de dados" } \\
\text { (1991); "Mais que Sorte... Um processo de raciocínio" (1994); "Corrente } \\
\text { Crítica" (1998) }\end{array}$ \\
\hline Tese Fundamental & $\begin{array}{l}\text { Em toda organização existe pelo menos uma restrição (gargalo) que } \\
\text { limita o seu desempenho. }\end{array}$ \\
\hline Meta da empresa & Fazer dinheiro agora e no futuro. \\
\hline Tipos de recursos & $\begin{array}{c}\text { Recurso gargalo, recursos não gargalos e recursos com capacidade } \\
\text { restrita }\end{array}$ \\
\hline $\begin{array}{l}\text { Conceito de gargalo ou } \\
\text { restrição }\end{array}$ & $\begin{array}{c}\text { É um recurso cuja capacidade é igual ou menor que a demanda } \\
\text { existente. }\end{array}$ \\
\hline $\begin{array}{l}\text { Tipos de restrições ou } \\
\text { gargalos }\end{array}$ & $\begin{array}{l}\text { Restrições internas (políticas de gerenciamento) e externas (que estão } \\
\text { fora do controle da organização). }\end{array}$ \\
\hline Medidas operacionais & Ganho (Throughput), Inventário e Despesas Operacionais. \\
\hline $\begin{array}{l}\text { Conceito de Ganho } \\
\text { (Throughput) }\end{array}$ & É dado pela diferença entre a receita obtida e o material direto utilizado. \\
\hline Conceito de Inventário & $\begin{array}{l}\text { Todo dinheiro que a organização investe na aquisição de coisas que ela } \\
\text { pretende vender. }\end{array}$ \\
\hline $\begin{array}{l}\text { Conceito de Despesas } \\
\text { Operacionais }\end{array}$ & $\begin{array}{c}\text { Todo dinheiro que a organização gasta para transformar o inventário em } \\
\text { ganho. }\end{array}$ \\
\hline Principal limitação & Foco no curto prazo \\
\hline Principais métodos & Processo de Raciocínio e metodologia Tambor-pulmão-corda. \\
\hline $\begin{array}{l}\text { Focalização das } \\
\text { decisões }\end{array}$ & $\begin{array}{c}\text { Dá-se por meio da identificação das restrições do sistema e da decisão } \\
\text { de como explorar tais restrições. }\end{array}$ \\
\hline Passos de Focalização & $\begin{array}{l}\text { 1. Identificar a (s) restrição (ões) do sistema; } \\
\text { 2. Decidir como explorar a (s) restrição (ões) do sistema; } \\
\text { 3. Subordinar tudo o mais à decisão do } 2^{\circ} \text { passo; } \\
\text { 4. Levantar a (s) restrição (ões) do sistema; } \\
\text { 5. Se, nos passos anteriores, uma restrição foi quebrada, voltar ao } 1^{\circ} \\
\text { passo, mas não deixar que a inércia se torne uma restrição no sistema. }\end{array}$ \\
\hline Principal Van & $\begin{array}{c}\text { Sua filosofia de gerenciamento considera as restrições que existem em } \\
\text { todos os sistemas, sejam eles empresariais ou não. }\end{array}$ \\
\hline
\end{tabular}

Quadro 1 - Resumo das principais características e conceitos da Teoria das Restrições Fonte: Elaboração própria a partir de MacArthur (1993), Guerreiro (1996), González (1999) e Cogan (2007).

\subsection{Bibliometria}

As diversas áreas do saber, em situação de maior ou menor desenvolvimento, passam por um processo de avaliação do conhecimento produzido, o que não é recente 
no ambiente acadêmico. Dentre as várias óticas sob as quais se podem avaliar aspectos relativos à produção científica, tais como: a metodológica, a epistemológica, a teórica, a paradigmática, a técnica, a bibliométrica e outras, Campos (2003 apud LEITE FILHO, 2006) assegura que os indicadores bibliométricos têm a prerrogativa de avaliar periódicos, textos científicos ou até mesmo a produção científica de uma área específica ou de um grupo de pesquisadores.

Como conceitua Tague-Sutckiffe (1992 apud MACIAS-CHAPULA, 1998, p. 134),

Bibliometria é o estudo dos aspectos quantitativos da produção, da disseminação e do uso da informação registrada. Usada pela primeira vez por Pritchard, em 1969, a bibliometria desenvolve padrões e modelos matemáticos para medir esses processos, usando seus resultados para elaborar previsões e apoiar tomadas de decisão.

Como é possível notar, trata-se de um conceito voltado a esclarecer os objetivos da disciplina, ou seja, medir quantitativamente determinados aspectos da produção científica.

Carrizo-Sainero (2007) apresenta três hipóteses que são necessárias para formular o conceito de Bibliometria, a saber: A bibliometria (i) encontra suas raízes epistemológicas na produção bibliográfica e, como aplicação desta, (ii) constitui um fator de mensuração e avaliação de fontes de informação, (iii) consistindo num método ou conjunto de métodos que podem ser empregados para avaliar a pesquisa.

Guedes e Borschiver (2005, p. 15) tratam a bibliometria como uma ferramenta estatística capaz de mapear e gerar diversos indicadores de produtividade, de tratamento e gestão da informação e do conhecimento, sobretudo em sistemas de informação e de comunicação.

Vanti (2002) e Guedes e Borschiver (2005) confirmam o caráter essencialmente quantitativo dos estudos bibliométricos em seus conceitos e acrescentam que os resultados obtidos funcionam como ferramenta para análise do comportamento dos pesquisadores na elaboração da produção científica, além de contribuir no processo decisorial de gestão da informação e do conhecimento. 
Os estudiosos da ciência da informação desenvolveram algumas metodologias específicas para realização da análise bibliométrica da produção científica. Essas metodologias ficaram conhecidas como "leis bibliométricas" e as principais, segundo Tague-Sutckiffe (1992 apud VANTI, 2002, p. 153), são:

a) Lei de Lotka ou Lei do Quadrado Inverso - tem foco na mensuração da produtividade dos pesquisadores, tendo por base um modelo de distribuição tamanho-freqüência dos vários pesquisadores em um conjunto de documentos. Essa lei considera que alguns pesquisadores, supostamente de maior prestígio em uma determinada área do conhecimento, produzem muito e muitos pesquisadores, supostamente de menor prestígio, produzem pouco.

b) Lei de Bradford ou Lei de Dispersão - relaciona-se com a dispersão da literatura periódica científica, por meio da avaliação da produtividade dos periódicos e do estabelecimento de núcleos e áreas de dispersão sobre um dado assunto em um mesmo grupo de periódicos.

c) Leis de Zipf ou Lei do Mínimo Esforço - referem-se à mensuração da freqüência de ocorrência de palavras em um determinado texto, que gera uma lista ordenada de termos de uma disciplina ou assunto e enseja a indexação temática automática.

Guedes e Borschiver (2005) destacam algumas aplicações práticas dessas leis. A Lei de Lotka se aplica principalmente na avaliação da produtividade de pesquisadores e no reconhecimento de centros de pesquisa mais desenvolvidos. A Lei de Bradford encontra utilidade no desenvolvimento de políticas de aquisição e descarte de periódicos em sistemas de gestão da informação. Por sua vez, as Leis de Zipf vêm sendo utilizadas na identificação de estilos distintos de autores e na elaboração de textos científicos e técnicos.

Além das leis bibliométricas já enumeradas, Guedes e Borschiver (2005) citam ainda os estudos baseados na Análise de Citações. Essa metodologia considera que a citação é um indicador relevante de influência de um trabalho em relação a outros, o que pode revelar algumas vinculações intelectuais. A Análise de Citações permite ainda 
identificar diversas outras características das referências bibliográficas que serviram de base para a elaboração de textos científicos e técnicos.

\section{METODOLOGIA}

Quanto aos objetivos, a pesquisa é classificada como descritiva. Esse tipo de pesquisa preocupa-se em observar os fatos, registrá-los, analisá-los, classificá-los e interpretá-los, sem a interferência do pesquisador (ANDRADE, 2002).

Em relação aos procedimentos, trata-se de uma pesquisa do tipo levantamento. Segundo Martins e Theóphilo (2007), os levantamentos costumam ser utilizados quando se pretende responder questões relativas à distribuição de uma variável ou relações entre características de elementos em suas condições normais. Este meio foi adotado em decorrência do objeto do estudo, que são os artigos científicos apresentados no Congresso Brasileiro de Custos acerca do tema "Teoria das Restrições".

Para a execução do presente estudo, foi considerado como universo de pesquisa o total dos trabalhos apresentados em todas as edições do Congresso Brasileiro de Custos (CBC) realizados no período de 1994 a 2008.

O Congresso Brasileiro de Custos é uma iniciativa da Associação Brasileira de Custos ( $A B C$ ), uma entidade de caráter científico, fundada em 1994, que agrupa pesquisadores e profissionais de diversas áreas do conhecimento (Contabilidade, Administração, Economia, Engenharia, Qualidade, entre outras) que estudam a gestão de custos sob uma ótica interdisciplinar. $A$ ABC tem como objetivo o desenvolvimento de conhecimentos e a aplicação de sistemas de gestão estratégica de custos, que contribuam para a melhoria da qualidade de produtos, flexibilidade de processos, redução de lead time e de custos, das organizações que desejam tornar-se competitivas em nível global (ABC, 2009).

De 1994 a 2008 foram realizadas 15 edições desse congresso, sendo que até o ano de 1999 o mesmo era denominado de Congresso Brasileiro de Gestão Estratégica de Custos. Além disso, em duas edições (1995 e 2005) o congresso foi realizado em 
paralelo com o Congresso Internacional de Custos. As regiões do Brasil que sediaram o Congresso Brasileiro de Custos foram: Sudeste (seis edições), Sul (cinco edições) e Nordeste (quatro edições). As regiões Centro-Oeste e Norte ainda não foram contempladas com a realização do CBC.

Os artigos foram capturados no portal da Associação Brasileira de Custos (http://www.abcustos.org.br), cuja base disponibiliza ao público em geral o título, autores e resumo dos trabalhos e o texto completo apenas para os associados. Os artigos foram selecionados por meio da opção de busca disponibilizada no site, a partir das seguintes palavras-chaves: Teoria das Restrições, Theory of Constraints e TOC. Foram localizados 48 artigos, porém um foi excluído porque, apesar de conter a expressão Teoria das Restrições, abordava uma temática totalmente diversa da escolhida para a presente investigação.

A Tabela 1 apresenta as edições do congresso e os respectivos anos de realização, cidades, Estados e instituições organizadoras do evento e a respectiva quantidade de trabalhos acerca do tema Teoria das Restrições.

Tabela 1 - Amostra e período pesquisado

\begin{tabular}{|c|c|c|c|c|c|}
\hline \multirow{2}{*}{ Edição } & & \multicolumn{2}{|c|}{ Sede do Congresso } & Quantidade & \\
\cline { 3 - 4 } de & Cidade/UF & Organização & trabalhos & $\%$ \\
\hline II & 1994 & São Leopoldo/RS & UNISINOS & 1 & $2,1 \%$ \\
\hline II & 1995 & Campinas/SP & UNICAMP & 1 & $2,1 \%$ \\
\hline III & 1996 & Curitiba/PR & UFPR & 0 & $0,0 \%$ \\
\hline IV & 1997 & Belo Horizonte/MG & PUC-MINAS & 3 & $6,4 \%$ \\
\hline V & 1998 & Fortaleza/CE & UFC & 3 & $6,4 \%$ \\
\hline VI & 1999 & São Paulo/SP & FEA/USP & 6 & $12,8 \%$ \\
\hline VII & 2000 & Recife/PE & UFPE & 4 & $8,5 \%$ \\
\hline VIII & 2001 & São Leopoldo/RS & UNISINOS & 2 & $4,3 \%$ \\
\hline IX & 2002 & São Paulo/SP & FECAP & 0 & $0,0 \%$ \\
\hline X & 2003 & Guarapari/ES & Diversas & 3 & $6,4 \%$ \\
\hline XI & 2004 & Porto Seguro/BA & FVC & 5 & $10,6 \%$ \\
\hline XII & 2005 & Florianópolis/SC & ABC & $\mathbf{1 1}$ & $\mathbf{2 3 , 4 \%}$ \\
\hline XIII & 2006 & Belo Horizonte/MG & ABC & 2 & $4,3 \%$ \\
\hline XIV & 2007 & João Pessoa/PB & ABC/UFPB & 3 & $6,4 \%$ \\
\hline XV & 2008 & Curitiba/PR & ABC/UFPR & 3 & $6,4 \%$ \\
\hline Total & & \multicolumn{5}{|l|}{} & & 47 & $100 \%$ \\
\hline
\end{tabular}

Fonte: Dados da pesquisa, Jun./2009 
A partir da tabela 1, verifica-se que a discussão em torno da Teoria das Restrições esteve presente em grande parte das edições do Congresso Brasileiro de Custos. Em 15 edições, 13 apresentaram trabalhos acerca do tema, ficando excluídos apenas os anos de 1996 e 2002. O CBC realizado em Florianópolis no ano de 2005 concentrou o maior número de trabalhos sobre a TOC, com 11 observações $(23,4 \%)$, seguido dos congressos de São Paulo (1999), com seis trabalhos $(12,8 \%)$ e Porto Seguro (2004), com cinco (10,6\%).

Foi desenvolvido um banco de dados com a utilização do Microsoft Excel 2007@ para registro dos dados coletados. O formulário do banco de dados foi composto por três partes: (1) informações gerais; (2) metodologia; e (3) categorias referenciais. A Parte 1 contemplou as seguintes variáveis: edição do evento, vínculo institucional dos autores, Estado da federação, quantidade de autores, nomes dos autores, sexo e enfoque temático. A Parte 2 foi composta pelas variáveis: tipologia da pesquisa quanto aos objetivos e procedimentos. A Parte 3 englobou as seguintes categorias referenciais: livros, periódicos, teses e dissertações, anais de eventos científicos, jornais e revistas, sites da WEB e outras categorias.

O item "enfoque temático" da Parte 1 foi categorizado nas seguintes áreas: Revisões teóricas sobre a TOC; Processo de Raciocínio da TOC; Estudos Comparativos entre a TOC e outras teorias/metodologias; Estudos Integração da TOC com outras teorias/metodologias; Aplicações gerais da TOC; Gestão de custos através da TOC; Medidas de Desempenho da TOC. A classificação se deu por meio da leitura e análise do título, do resumo e do artigo.

No item "tipologia da pesquisa quanto aos objetivos" da Parte 2 foram considerados os seguintes tipos: descritiva, explicativa, exploratória e exclusivamente teórica, conforme tipologia proposta por Gil (1999). O item "procedimentos" da Parte 2 foi composto pelas seguintes categorias: bibliográfica; documental; experimental; levantamento (ou pesquisa de campo); estudo de caso; e pesquisa operacional, de acordo com os procedimentos citados por Martins e Theóphilo (2007). Para fins de classificação foram analisados o resumo, a introdução e/ou a parte do artigo que tratava 
da metodologia do trabalho. Os artigos que utilizavam mais de um procedimento foram classificados pelo que demonstrava ser o principal.

\section{RESULTADOS DA PESQUISA}

Em consonância com os objetivos principal e secundário da pesquisa, são apresentados nessa seção alguns aspectos da produção científica analisada, como: quantidade de autores por artigo, gênero, Estado e vinculação institucional dos autores, tipos de pesquisa, procedimentos metodológicos e enfoques temáticos dos trabalhos e medidas descritivas das categorias referenciais utilizadas nos artigos analisados.

A Tabela 2 apresenta as freqüências absolutas e relativas da quantidade de autores por artigo e a média de autores por trabalho.

Os dados revelam uma quantidade máxima de cinco autores por artigo. A maior parte dos trabalhos $(31,9 \%)$ foi assinada por dois autores. Os artigos com até três autores representam $83 \%$ da amostra analisada. De maneira geral, a média de autores por artigo é 2,32. Leite Filho (2006) e Braga, Cruz e Oliveira (2007) constataram que nos anais de congressos, tanto os voltados para pesquisadores profissionais, quanto em nível de iniciação científica, as maiores freqüências referem-se a trabalhos com dois autores. Os resultados da presente pesquisa são totalmente compatíveis com a constatação desses autores.

Tabela 2 - Quantidade de autores

\begin{tabular}{|c|c|c|c|}
\hline Quantidade de Autores & $\mathbf{F}$ & $\boldsymbol{f}$ & fac \\
\hline 1 autor & 13 & $27,7 \%$ & $27,7 \%$ \\
\hline 2 autores & $\mathbf{1 5}$ & $\mathbf{3 1 , 9} \%$ & $59,6 \%$ \\
\hline 3 autores & 11 & $23,4 \%$ & $\mathbf{8 3 , 0} \%$ \\
\hline 4 autores & 7 & $14,9 \%$ & $97,9 \%$ \\
\hline 5 autores & 1 & $2,1 \%$ & $100,0 \%$ \\
\hline Total de artigos & 47 & $100,0 \%$ & \\
\hline Total de autores & 109 & & \\
\hline Média de autores/artigo & 2,32 & & \\
\hline
\end{tabular}

Fonte: Dados da pesquisa, Jun./2009 
A Tabela 3 evidencia o gênero dos autores dos artigos com abordagem na Teoria das Restrições apresentados no Congresso Brasileiro de Custos.

Tabela 3 - Gênero dos autores

\begin{tabular}{|c|c|c|}
\hline Sexo & $\mathbf{F}$ & $\boldsymbol{f}$ \\
\hline Feminino & 28 & $25,7 \%$ \\
\hline Masculino & 81 & $74,3 \%$ \\
\hline Total & 109 & $100,0 \%$ \\
\hline
\end{tabular}

Fonte: Dados da pesquisa, Jun./2009

Os dados mostram que a autoria dos artigos sobre o tema Teoria das Restrições apresentados no Congresso Brasileiro de Custos em sua maioria é do sexo masculino (74,3\%), enquanto que o gênero feminino concentra $25,7 \%$ dos artigos sobre o tema. Silva, Oliveira e Ribeiro (2005) e Leite Filho (2006) também investigaram o gênero dos autores dos artigos analisados em suas pesquisas. Concluíram que $30,65 \%$ e $29,2 \%$ dos autores dos trabalhos analisados eram do gênero feminino e $69,35 \%$ e $70,8 \%$ do masculino, respectivamente. Pode-se notar que os resultados da presente pesquisa são compatíveis com a constatação desses autores.

A Tabela 4 apresenta os Estados do Brasil declarados como de origem dos autores dos artigos com abordagem na Teoria das Restrições apresentados no Congresso Brasileiro de Custos.

A Tabela 5 evidencia as regiões do Brasil em que se localizam os autores dos artigos com abordagem na Teoria das Restrições apresentados no Congresso Brasileiro de Custos. 
Teoria das Restrições: Um Estudo Bibliométrico da Produção Científica Apresentada no Congresso Brasileiro de Custos (1994-2008) Claudia Cruz, Alessandra Marques, Roberval Silva, Samuel Cogan

Tabela 4 - Estado dos autores dos artigos

\begin{tabular}{|c|c|c|c|}
\hline Estado & F & $\boldsymbol{f}$ & fac \\
\hline São Paulo (SP) & 15,5 & $33,0 \%$ & $33,0 \%$ \\
\hline Rio de Janeiro (RJ) & 11,7 & $24,9 \%$ & $57,9 \%$ \\
\hline Santa Catarina (SC) & 7 & $14,9 \%$ & $72,8 \%$ \\
\hline Rio Grande do Sul (RS) & 4 & $8,5 \%$ & $81,3 \%$ \\
\hline Minas Gerais (MG) & 2,8 & $6,0 \%$ & $87,2 \%$ \\
\hline Paraná (PR) & 1,5 & $3,2 \%$ & $90,4 \%$ \\
\hline Rio Grande do Norte (RN) & 1 & $2,1 \%$ & $92,6 \%$ \\
\hline Espírito Santo (ES) & 1 & $2,1 \%$ & $94,7 \%$ \\
\hline Pará (PA) & 1 & $2,1 \%$ & $96,8 \%$ \\
\hline Outros países/Não identificado & 1,5 & $3,2 \%$ & $100 \%$ \\
\hline Total & 47 & $100 \%$ & \\
\hline
\end{tabular}

Fonte: Dados da pesquisa, Jun./2009

Tabela 5 - Região dos autores dos artigos

\begin{tabular}{|c|c|c|c|}
\hline Região & F & $\boldsymbol{f}$ & fac \\
\hline Sudeste & 31 & $66,0 \%$ & $66,0 \%$ \\
\hline Sul & 12,5 & $26,6 \%$ & $92,6 \%$ \\
\hline Nordeste & 1 & $2,1 \%$ & $94,7 \%$ \\
\hline Norte & 1 & $2,1 \%$ & $96,8 \%$ \\
\hline Centro-Oeste & 0 & $0,0 \%$ & $96,8 \%$ \\
\hline Outros países/Não identificado & 1,5 & $3,2 \%$ & $100 \%$ \\
\hline Total & 47 & $100 \%$ & \\
\hline
\end{tabular}

Fonte: Dados da pesquisa, Jun./2009

As tabelas 4 e 5 apresentam os artigos de acordo com o Estado de origem dos seus autores e as respectivas regiões. Para a classificação por Estado, quando um mesmo artigo possuía autores de estados diferentes, a pontuação do artigo era dividida proporcionalmente ao número de autores de cada estado. O Estado com a maior participação no total de artigos foi São Paulo, com 33\%, seguido do Rio de Janeiro $(24,9 \%)$ e Santa Catarina (14,9\%). Embora os artigos com autores de apenas três estados tenham representado $72,8 \%$ da amostra pesquisa, verificou-se que nenhum Estado apresentou regularidade em todas as edições do CBC analisadas, sendo os estados de São Paulo e Rio de Janeiro os mais regulares. Dos 27 estados da federação, autores de apenas nove tiveram trabalhos com abordagem da Teoria das Restrições nas edições analisadas do Congresso Brasileiro de Custos. Isso revela uma concentração das pesquisas, sobretudo nos Estado de São Paulo e Rio de Janeiro. 
A análise dos artigos de acordo com a região de origem dos autores permite verificar a concentração das pesquisas na área de Teoria das Restrições nos estados das Regiões Sul e Sudeste (92,6\% dos trabalhos). As regiões Norte e Nordeste estão representadas em um trabalho cada e a região Centro-Oeste não figurou em nenhum dos artigos da amostra. Houve ainda um trabalho em que um dos autores era vinculado a uma instituição colombiana e outro cujo vínculo e origem não foram identificados.

A Tabela 6 evidencia as freqüências absolutas e relativas dos artigos por vínculo institucional declarado pelos autores. Nos casos em que um artigo possuísse autores de instituições diferentes, a pontuação do artigo foi dividida proporcionalmente pela quantidade de instituições declaradas e foi atribuída a cada uma dessas as respectivas participações.

Tabela 6 - Vínculo institucional dos autores

\begin{tabular}{|c|c|c|c|}
\hline Vínculo Institucional & F & $\boldsymbol{f}$ & fac \\
\hline Universidade Federal do Rio de Janeiro (UFRJ) & 10,7 & $22,8 \%$ & $22,8 \%$ \\
\hline Universidade de São Paulo (USP) & 6,8 & $14,5 \%$ & $37,2 \%$ \\
\hline Universidade do Vale do Rio dos Sinos (UNISINOS) & 4 & $8,5 \%$ & $45,7 \%$ \\
\hline Universidade Comunitária Regional de Chapecó (UNOCHAPECO) & 4 & $8,5 \%$ & $54,3 \%$ \\
\hline Escola de Administração de Empresas de São Paulo (EAESP-FGV) & 2,7 & $5,7 \%$ & $60,0 \%$ \\
\hline Universidade Federal de Santa Catarina (UFSC) & 2,5 & $5,3 \%$ & $65,3 \%$ \\
\hline Fundação Escola de Comércio Álvares Penteado (FECAP) & 1,5 & $3,2 \%$ & $68,5 \%$ \\
\hline Vínculos não acadêmicos & 1,7 & $3,6 \%$ & $72,1 \%$ \\
\hline Outras instituições acadêmicas (19) & 13,1 & $27,9 \%$ & $100 \%$ \\
\hline Total & 47 & $100 \%$ & \\
\hline
\end{tabular}

Fonte: Dados da pesquisa, Jun./2009

Os vínculos institucionais declarados pelos autores dos trabalhos apontam para um total de 26 instituições acadêmicas e duas organizações empresariais. Entre as instituições acadêmicas, a Universidade Federal do Rio de Janeiro foi a que mais figurou entre os artigos analisados nesse trabalho, com $22,8 \%$, destacando que todos os autores vinculados a essa instituição estão ligados à Faculdade de Administração e Ciências Contábeis (FACC/UFRJ) da referida instituição; em seguida a Universidade de São Paulo, com $14,5 \%$, sendo que no caso da USP incluem-se trabalhos de autores da Escola de Engenharia de São Carlos (EESC/USP), Escola Politécnica (POLI/USP) e Faculdade de Administração, Economia e Contabilidade (FEA/USP). Destaca-se, ainda, 
a UNISINOS e a UNOCHAPECO, como $8,5 \%$ dos trabalhos cada uma. Ressalta-se também que 19 instituições acadêmicas apareceram apenas uma vez nos artigos dentre as 13 edições do CBC pesquisadas, indicando falta de continuidade das pesquisas no tema Teoria das Restrições.

A autoria de $54,3 \%$ dos artigos está concentrada em autores de apenas quatro instituições, todas das regiões Sul e Sudeste do Brasil, onde se localiza a maioria dos programas de pós-graduação Stricto Sensu em Administração e Ciências Contábeis, área de formação da maioria dos autores. Resultados parecidos foram encontrados por Cardoso et al. (2005) e Leite Filho (2006), em que também se verificou elevado grau de concentração das publicações em autores de poucas instituições.

A tabela 7 apresenta os autores que mais desenvolveram pesquisas sobre 0 tema Teoria das Restrições que foram apresentadas no Congresso Brasileiro de Custos, a quantidade de trabalhos e o número das edições em que tiveram publicação. Destaca-se que para quantificar o número de trabalhos de cada autor não se fez distinção entre autoria e co-autoria.

Tabela 7 - Autores mais produtivos

\begin{tabular}{|c|c|c|}
\hline Autor(a) & № de trabalhos & № de Edições \\
\hline Samuel Cogan & 10 & 7 \\
\hline Joanília Neide de Sales Cia & 5 & 4 \\
\hline Anselmo Rocha Neto & 4 & 4 \\
\hline Josilmar Cordenonssi Cia & 3 & 2 \\
\hline Antonio Cesar Bornia & 2 & 2 \\
\hline Patricia González González & 2 & 2 \\
\hline Wilson Kendy Tachibana & 2 & 1 \\
\hline José Antonio Valle Antunes Júnior & 2 & 2 \\
\hline Adão Marcos Siqueira & 2 & 2 \\
\hline Vilmar Oenning & 2 & 2 \\
\hline José Augusto Veiga da Costa Marques & 2 & 1 \\
\hline Demais autores (73) & 1 & 1 \\
\hline
\end{tabular}

Fonte: Dados da pesquisa, Jun./2009

Entre os autores com mais trabalhos sobre Teoria das Restrições publicados nos anais do Congresso Brasileiro de Custos, estão: Samuel Cogan (FACC/UFRJ) - 10 trabalhos/ 7 edições; Joanília Neide de Sales Cia (EAESP/FGV e FEA/USP) - 5 trabalhos/ 4 edições; Anselmo Rocha Neto (UNOCHAPECO) - 4 trabalhos/ 4 edições; 
Josilmar Cordenonssi Cia (EAESP-FGV) - 3 trabalhos/ 2 edições; outros sete autores tiveram participação em dois trabalhos cada. $O$ indicativo da falta de continuidade da produção científica na área de Teoria das Restrições já apontada na tabela 6 é corroborado quando se verifica que 73 autores tiveram participação em apenas um trabalho durante os 15 anos do Congresso Brasileiro de Custos.

A partir do estudo das leis bibliométricas, Guedes e Borschiver (2005) destacam a Lei de Lotka, cujo principal objetivo é estimar o grau de relevância de autores, em dada área do conhecimento. A premissa básica da Lei de Lotka de que alguns pesquisadores, supostamente de maior prestígio em uma determinada área do conhecimento, produzem muito e muitos pesquisadores, supostamente de menor prestígio, produzem pouco, se confirma para a publicação de artigos sobre o tema Teoria das Restrições nas edições pesquisadas do Congresso Brasileiro de Custos, de acordo com os dados da Tabela 7.

A tabela 8 apresenta as freqüências absolutas e relativas dos enfoques temáticos dos artigos sobre Teoria das Restrições apresentados no Congresso Brasileiro de Custos.

Tabela 8 - Enfoque temático dos artigos

\begin{tabular}{|c|c|c|}
\hline Enfoque Temático & $\mathbf{F}$ & $\boldsymbol{f}$ \\
\hline Aplicações Gerais da TOC & 8 & $17,0 \%$ \\
\hline Estudos Comparativos da TOC & $\mathbf{1 5}$ & $\mathbf{3 1 , 9 \%}$ \\
\hline Estudos de Integração TOC & $\mathbf{1 3}$ & $\mathbf{2 7 , 7 \%}$ \\
\hline Gestão de Custos através da TOC & 3 & $6,4 \%$ \\
\hline Medidas de Desempenho da TOC & 2 & $4,3 \%$ \\
\hline Processo de Raciocínio da TOC & 5 & $10,6 \%$ \\
\hline Revisões teóricas da TOC & 1 & $2,1 \%$ \\
\hline Total & 47 & $100 \%$ \\
\hline
\end{tabular}

Fonte: Dados da pesquisa, Jun./2009

Dos enfoques adotados nos trabalhos sobre a Teoria das Restrições apresentados no $\mathrm{CBC}$, a maioria $(31,9 \%)$ tratou-se de estudos cuja finalidade era estabelecer comparações entre a referida teoria e outros métodos de gestão e controle de custos, principalmente o Activity Based Costing ( $A B C)$, o custo variável, custeio por absorção, custo padrão entre outros; esses estudos tinham como finalidade apresentar 
as vantagens e desvantagens da TOC em relação a esses métodos. Em seguida, aparecem os estudos que discutiram a integração da TOC com outros métodos de gestão e controle de custos, com 27,7\% (13 observações) dos trabalhos; os artigos com esse enfoque buscaram destacar como as premissas da TOC podem contribuir para o aperfeiçoamento de outros métodos. Os textos que trataram, por meio de discussões teóricas ou de estudos de caso, das aplicações gerais da TOC representam 17\% (oito trabalhos) da amostra pesquisada. Destaca-se ainda o enfoque no processo de raciocínio na TOC que foi objeto de cinco artigos (10,6\%).

Apesar da recente expansão das discussões em torno da Teoria das Restrições, os dados da tabela 8 permitem constatar que trabalhos com abordagem nessa teoria estão sendo elaborados sob uma quantidade considerável de enfoques. Isso aponta para a potencialidade dessa teoria para a realização de pesquisas, principalmente as que se valem de dados empíricos para testar as premissas que caracterizam a TOC.

A tabela 9 apresenta as freqüências das estratégias de pesquisa adotadas nos artigos sobre a TOC apresentados no CBC de acordo com o enfoque temático.

Tabela 9 - Enfoque temático Vs. Estratégia da pesquisa

\begin{tabular}{|c|c|c|c|c|c|}
\hline & \multicolumn{4}{|c|}{ Estratégias de Pesquisa } & \\
\cline { 2 - 5 } Enfoque Temático & Bibliográfica & $\begin{array}{c}\text { Estudo } \\
\text { de Caso }\end{array}$ & $\begin{array}{c}\text { Pesquisa } \\
\text { de Campo }\end{array}$ & $\begin{array}{c}\text { Pesquisa } \\
\text { Operaciona } \\
\text { I }\end{array}$ & Total \\
\hline Aplicações Gerais da TOC & 2 & $\mathbf{5}$ & 0 & 1 & 8 \\
\hline Estudos Comparativos da TOC & $\mathbf{1 1}$ & 2 & 0 & 2 & 15 \\
\hline Estudos de Integração TOC & 6 & 3 & 0 & $\mathbf{4}$ & 13 \\
\hline $\begin{array}{c}\text { Gestão de Custos através da } \\
\text { TOC }\end{array}$ & 1 & 1 & 0 & 1 & 3 \\
\hline $\begin{array}{c}\text { Medidas de Desempenho da } \\
\text { TOC }\end{array}$ & 0 & 2 & 0 & 0 & 2 \\
\hline Processo de Raciocínio da TOC & 0 & 3 & $\mathbf{1}$ & 1 & 5 \\
\hline Revisões teóricas da TOC & 1 & 0 & 0 & 0 & 1 \\
\hline Total & 21 & 16 & 1 & 9 & 47 \\
\hline Percentual & $44,7 \%$ & $34,0 \%$ & $2,1 \%$ & $19,2 \%$ & $100 \%$ \\
\hline
\end{tabular}

Fonte: Dados da pesquisa, Jun./2009.

Dos procedimentos de pesquisa adotados nos trabalhos sobre TOC apresentados no CBC, 44,7\% tratavam-se de pesquisas bibliográficas, ou seja, revisões teóricas sobre o conceito e as premissas da Teoria das Restrições, suas vantagens e 
desvantagens. Em seguida aparecem os estudos de caso, que foram 16, o que representa $34 \%$ do total. Foi observado apenas um trabalho cuja estratégia de pesquisa foi classificada como pesquisa de campo ou levantamento. A pesquisa operacional, metodologia que utiliza modelos para estruturar processos de tomada de decisão aparentemente não estruturados (EHRLICH, 1991, p. 13) foi identificada em nove artigos (19,2\%), sobretudo com a técnica de modelos quantitativos simulados.

Quando relacionadas com os enfoques temáticos, verifica-se que a maioria das pesquisas bibliográficas abordou estudos comparativos da TOC com outros métodos, ou seja, esses estudos utilizaram apenas fontes teóricas para sustentar os argumentos expostos. Já a maioria dos estudos de caso eram aplicações gerais da TOC em setores específicos, tais como indústria, comércio e, principalmente, prestação de serviços. Destaca-se a tentativa de estudar a aplicação da TOC em outro ramo que não o industrial, uma vez que a teoria foi desenvolvida na realidade do ambiente fabril. Os modelos desenvolvidos nas pesquisas operacionais, em sua maioria, procuraram simular a aplicação da integração das premissas da TOC com outros métodos de gestão e controle de custos.

A tabela 10 apresenta as freqüências das tipologias de pesquisa quanto aos objetivos adotadas nos artigos sobre a TOC apresentados no CBC e de acordo com o enfoque temático.

Tabela 10 - Enfoque temático Vs. Tipologia da pesquisa quanto aos objetivos

\begin{tabular}{|l|r|r|r|r|}
\hline & \multicolumn{2}{|c|}{ Tipologia quanto aos objetivos da } & \\
\cline { 2 - 4 } \multicolumn{1}{c|}{ Enfoque Temático } & pesquisa & \multicolumn{1}{c|}{} \\
\cline { 2 - 5 } & Descritiva & Exploratória & $\begin{array}{c}\text { Exclusivamente } \\
\text { teórica }\end{array}$ & \multicolumn{1}{c|}{ Total } \\
\hline Aplicações Gerais da TOC & 5 & 0 & 3 & 8 \\
\hline Estudos Comparativos da TOC & 3 & 1 & $\mathbf{1 1}$ & 15 \\
\hline Estudos de Integração TOC & $\mathbf{7}$ & 1 & $\mathbf{5}$ & 13 \\
\hline Gestão de Custos através da TOC & 1 & 1 & 1 & 3 \\
\hline Medidas de Desempenho da TOC & 2 & 0 & 0 & 2 \\
\hline Processo de Raciocínio da TOC & 3 & 2 & 0 & 5 \\
\hline Revisões teóricas da TOC & 0 & 0 & 1 & 1 \\
\hline Total & 21 & 5 & 21 & 47 \\
\hline Percentual & $44,7 \%$ & $10,6 \%$ & $44,7 \%$ & $100 \%$ \\
\hline
\end{tabular}

Fonte: Dados da pesquisa, Jun./2009 
Quando da classificação dos trabalhos analisados quanto aos objetivos pretendidos, foram detectados basicamente três tipos de pesquisa: descritiva, exploratória e teórica. Em geral, os trabalhos sobre a TOC apresentados no CBC tinham objetivos teóricos ou descritivos, com $44,7 \%$ cada (21 trabalhos de finalidade exclusivamente teórica e 21 com o objetivo de descrever um fenômeno ou elemento). Os trabalhos de finalidade exploratória representaram $10,6 \%$ da amostra (5 observações). Esses resultados indicam um estágio inicial das pesquisas sobre o tema, uma vez que a maioria dos trabalhos se limita a apresentar as premissas teóricas da TOC ou descrever sua aplicação por meio de casos simulados. O tema ainda carece de investigações que expliquem sua adoção, bem como os impactos decorrentes.

Quando relacionadas com os enfoques temáticos, verifica-se que a maioria das pesquisas com abordagem exclusivamente teórica tratou de estudos comparativos da TOC com outros métodos, ou seja, foram estudos que se propuseram a consolidar as premissas teóricas da Teoria das Restrições a partir da sua comparação com outros métodos relacionados a gestão e controle de custos. Dos trabalhos com finalidade descritiva, sete foram estudos de integração da TOC com outros métodos. Os estudos com enfoque no processo de raciocínio da TOC tiveram abordagem descritiva (três) e exploratória (duas).

A tabela 11 apresenta as freqüências das categorias referenciais utilizadas nos artigos sobre a TOC apresentados no CBC por edição do evento e no geral. 
Teoria das Restrições: Um Estudo Bibliométrico da Produção Científica Apresentada no Congresso Brasileiro de Custos (1994-2008) Claudia Cruz, Alessandra Marques, Roberval Silva, Samuel Cogan

Tabela 11 - Categorias referenciais dos trabalhos

\begin{tabular}{|c|c|c|c|c|c|c|c|c|c|c|}
\hline Edição & $\begin{array}{c}\text { № de } \\
\text { Artigos }\end{array}$ & Livros & Periódicos & $\begin{array}{l}\text { Jornais/ } \\
\text { revistas }\end{array}$ & $\begin{array}{c}\text { Teses/ } \\
\text { Dissertações }\end{array}$ & Sites & Anais & Outros & Total & $\begin{array}{l}\text { Média/ } \\
\text { Edição }\end{array}$ \\
\hline $\mathrm{I}$ & 1 & 4 & 1 & 0 & 0 & 0 & 0 & 1 & 6 & 6,0 \\
\hline III & 1 & 10 & 0 & 0 & 0 & 0 & 0 & 0 & 10 & 10,0 \\
\hline IV & 3 & 22 & 4 & 0 & 2 & 0 & 2 & 1 & 31 & 10,3 \\
\hline $\mathrm{V}$ & 3 & 39 & 4 & 0 & 0 & 0 & 2 & 1 & 46 & 15,3 \\
\hline $\mathrm{VI}$ & 6 & 53 & 34 & 0 & 7 & 7 & 7 & 3 & 111 & 18,5 \\
\hline VII & 4 & 27 & 27 & 0 & 0 & 0 & 6 & 1 & 61 & 15,3 \\
\hline VIII & 2 & 14 & 6 & 0 & 2 & 0 & 5 & 0 & 27 & 13,5 \\
\hline$X$ & 3 & 23 & 13 & 0 & 1 & 0 & 3 & 1 & 41 & 13,7 \\
\hline $\mathrm{XI}$ & 5 & 33 & 17 & 0 & 3 & 1 & 3 & 3 & 60 & 12,0 \\
\hline XII & 11 & 121 & 68 & 2 & 9 & 9 & 14 & 8 & 231 & 21,0 \\
\hline XIII & 2 & 19 & 2 & 0 & 2 & 1 & 1 & 0 & 25 & 12,5 \\
\hline XIV & 3 & 22 & 13 & 0 & 7 & 2 & 5 & 0 & 49 & 16,3 \\
\hline $\mathrm{XV}$ & 3 & 24 & 2 & 0 & 7 & 5 & 2 & 2 & 42 & 14,0 \\
\hline Total & 47 & 411 & 191 & 2 & 40 & 25 & 50 & 21 & 740 & 15,7 \\
\hline$\%$ & $N D$ & $55,5 \%$ & $25,8 \%$ & $0,3 \%$ & $5,4 \%$ & $3,4 \%$ & $6,8 \%$ & $2,8 \%$ & $100 \%$ & $N D$ \\
\hline Média & $N D$ & 8,7 & 4,1 & 0,04 & 0,9 & 0,5 & 1,1 & 0,4 & 15,7 & $N D$ \\
\hline
\end{tabular}

Fonte: Dados da pesquisa, Jun./2009.

Dos 47 trabalhos analisados nessa pesquisa, foram observadas 740 referências. No geral, os trabalhos apresentam uma média de 15,7 referências. A edição que teve a maior média foi a XII $(21,0)$. Pode-se constatar que a categoria mais referenciada nos trabalhos analisados foi Livros, com 55,5\% das fontes utilizadas. Essa freqüência oscila pouco quando se analisa as edições individualmente. Essa categoria, no geral, apresenta uma média de 8,7, que representa a maior média em relação às outras categorias, além de ser a única presente em todos os trabalhos de todas as edições analisadas. Logo após tem-se as categorias Periódicos, Anais de Congressos e Teses e Dissertações, com $25,8 \%, 6,8 \%$ e $5,4 \%$, respectivamente.

A categoria Jornais/Revistas obteve a menor média $(0,04)$ e foi observada em apenas um dos trabalhos da amostra. Verifica-se que houve uma redução da categoria Livros nas últimas edições analisadas em relação às primeiras. De forma inversa, houve um aumento na categoria Periódicos nas últimas edições analisadas em relação às primeiras.

Pesquisas com objetivos semelhantes aos do presente estudo foram desenvolvidas por Cardoso et al. (2005), Silva, Oliveira e Ribeiro Filho (2005), Leite 
Filho (2006), Braga, Cruz e Oliveira (2007) e Schneider, Carneiro e Dutra (2009). Os resultados também são bastante parecidos, em que a bibliografia utilizada nos artigos mostra um posicionamento conservador e convencional dos autores na construção de seus referenciais bibliográficos. Os autores dos trabalhos analisados fazem poucas consultas a periódicos e, menos ainda, anais de congressos de natureza científica, segundo os dados da tabela 11. Esse fato, segundo Leite Filho (2006), dificulta as discussões teórico-empíricas contemporâneas, que revelam o 'estado da arte' do tema em discussão. Braga, Cruz e Oliveira (2007) afirmam que essa constatação se agrava quando se considera a facilidade de acesso a periódicos online por meio da Internet.

\section{CONCLUSÕES}

A presente pesquisa de natureza descritiva teve como objetivo principal analisar os trabalhos científicos com abordagem do tema "Teoria das Restrições", apresentados no Congresso Brasileiro de Custos (CBC) de 1994 a 2008, por meio do estudo bibliométrico. E teve como objetivo secundário explorar alguns aspectos da produção analisada, como: quantidade de autores, gênero, autores mais prolíficos, Estado de origem e vinculação institucional dos autores dos artigos, tipologia das pesquisas e procedimentos metodológicos utilizados nesses artigos, além dos enfoques temáticos adotados e referências utilizadas.

Os principais resultados da amostra de 47 artigos analisados revelam que: a maioria dos trabalhos tinha dois autores e ainda que $83 \%$ dos trabalhos eram de até três autores; em torno de dois terços dos autores são do sexo masculino; as regiões Sudeste e Sul são a origem da maioria dos autores e concentram $92,6 \%$ dos trabalhos, com destaque para os Estados do Rio de Janeiro e São Paulo; a Universidade Federal do Rio de Janeiro foi a instituição mais recorrente e os autores mais prolíficos, de um total de 84, foram: Samuel Cogan, Joanília Neide de Sales Cia e Anselmo Rocha Neto, o que confirma a Lei de Lotka que diz que alguns pesquisadores, supostamente de maior prestígio em uma determinada área do conhecimento, produzem muito e muitos pesquisadores, supostamente de menor prestígio, produzem pouco. Os enfoques mais 
abordados nos trabalhos foram os que buscaram comparar ou integrar as premissas da Teoria das Restrições com outros métodos de controle e gestão de custos. Não obstante o desenvolvimento recente da Teoria das Restrições, verificou-se que trabalhos em torno desse tema estão sendo elaborados sob uma quantidade considerável de enfoques. Isso indica a potencialidade do tema para a realização de pesquisas, sobretudo com base em dados empíricos para testar as premissas que caracterizam a TOC.

Quanto à metodologia adotada nos artigos investigados, a estratégia mais adotada foi pesquisa bibliográfica, seguida de estudo de caso; em sua maioria os trabalhos tiveram finalidade descritiva ou exclusivamente teórica. Em relação às referências, verificou-se uma média de 15,7 referências por artigo na amostra analisada; a categoria referencial mais citada foi Livros, com $55,5 \%$ do total de referências. Em seguida, tem-se: Periódicos (25,8\%), Anais de Congressos (6,8\%), Teses e Dissertações $(5,4 \%)$, Sites $(3,4 \%)$, Outras categorias $(2,8 \%)$ e Jornais e revistas $(0,3 \%)$.

Conclui-se que a considerável concentração das referências bibliográficas utilizadas na elaboração dos trabalhos em livros, as limitadas consultas a periódicos e as poucas ocorrências de sites, teses e dissertações e anais de congressos apontam para um posicionamento conservador e convencional dos autores dos trabalhos e podem configurar um estágio incipiente das pesquisas com abordagem no tema Teoria das Restrições. As considerações se limitam ao período e à amostra pesquisada. Como recomendação para futuras pesquisas, sugere-se a investigação de outros veículos de publicação que aceitem trabalhos relacionados ao tema abordado.

\section{REFERÊNCIAS}

ANDRADE, M. M. (2002). Como preparar trabalhos para cursos de pós-graduação: noções básicas. (5 ed.). São Paulo: Atlas, 154 p.

ASSOCIAÇÃO BRASILEIRA DE CUSTOS. Histórico e Objetivos. Disponível em: $<$ http://www.abcustos.org.br> Acesso em: 10/Jul/2009. 
BRAGA, J. P.; CRUZ, C. F.; OLIVEIRA, J. R. S. (2007). Pesquisa contábil no Nordeste: um estudo bibliométrico da produção científica apresentada no Encontro Regional de Estudantes de Ciências Contábeis. In: 4ํㅡㄹ Congresso USP de Iniciação Científica em Contabilidade, São Paulo. Anais...São Paulo, FEA/USP.

CARDOSO, R. L. et. al. (2005). Pesquisa científica em contabilidade entre 1990 e 2003. Revista de Administração de Empresas (RAE). São Paulo, 45(2): 34-45.

CARRIZO-SAINERO, G. Toward a concept of bibliometrics. Madrid: Universidad Carlos III.

<http://www.ucm.es/info/multidoc/publicaciones/journal/pdf/bibliometria-eng.pdf>. Acesso em: 24/jan/2009.

COGAN, S. (2007). Contabilidade gerencial: Uma abordagem da teoria das restrições. São Paulo: Saraiva, 294 p.

COGAN, S. (1999). Teoria das Restrições versus Custeio Baseado em Atividades: Um aparente conflito. In: VI Congresso Brasileiro de Custos, São Paulo, 1999. Anais...São Paulo, ABCustos.

EHRLICH, P. J. (1991). Pesquisa operacional: curso introdutório. São Paulo: Atlas, 190 p.

GIL, A. C. (1999). Métodos e técnicas de pesquisa social. (5 ed.). São Paulo: Atlas, 207 p.

GIUNTINI, N. et. al. (2002). Teoria das Restrições, uma nova forma de ver e pensar o gerenciamento empresarial. In: Seminário USP de Contabilidade, 2, São Paulo. Anais... São Paulo: FEA/USP.

GOLDRATT, Eliyahu M.; COX, Jeff. (1992). The Goal: A process of ongoing improvement. 2nd rev. edition North. River Press, 384 p.

GONZÁLEZ, P. G. (1999). Teoria das Restrições sob um enfoque de tomada de decisão e de mensuração econômica. In: VI Congresso Brasileiro de Custos, São Paulo. Anais...São Paulo, ABCustos.

GUEDES, V. L. S.; BORSCHIVER, S. (2005). Bibliometria: uma ferramenta estatística para a gestão da informação e do conhecimento, em sistemas de informação, de comunicação e de avaliação científica e tecnológica. In: CINFORM - Encontro Nacional de Ciência da Informação, VI. Salvador. Anais...Salvador, UFBA.

GUERREIRO, R. (1996). Os princípios da teoria das restrições sob a ótica da mensuração econômica. Caderno de Estudos - USP, São Paulo, 13(1):25-35. 
LEITE FILHO, G. A. (2006). Padrões de produtividade de autores em periódicos e congressos na área de contabilidade no Brasil: um estudo bibliométrico. In: Congresso USP de Controladoria e Contabilidade, 6, São Paulo. Anais...São Paulo: FEA/USP.

LINTER. Um passeio pela TOC... (2002). Disponível: <http://www.linter.com.br/pdf/intro_toc.pdf. Acesso em: 31/Jan/2009.

MACARTHUR, J. B. (1993). Theory of constraints and activity-based costing: Friends or foes? Journal of Cost Management, Florida, Summer: 50-56.

MACIAS-CHAPULA, C. A. (1998). O papel da informetria e da cienciometria e sua perspectiva nacional e internacional. Revista Ciência da Informação, Brasília, 27( 2):134-140.

MARTINS, G. A.; THEÓPHILO, C. R. (2007). Metodologia da investigação científica para ciências sociais aplicadas. São Paulo: Atlas, 225 p.

ROGERS, P.; REIS, E. A. (2005). Teoria das Restrições e decisões de longo prazo: caminho para a convergência. In: Congresso USP de Contabilidade e Controladoria, 5, São Paulo. Anais...São Paulo: FEA/USP.

SCHNEIDER, A. B.; CARNEIRO, M. L.; DUTRA, A. (2009). Análise da produção científica brasileira sobre avaliação de desempenho organizacional no período de 1997 a 2007. In: IV Encontro de Estudos em Estratégia, Recife. Anais..., Recife, ANPAD.

SILVA, A. C. B.; OLIVEIRA, E. C.; RIBEIRO FILHO, J. F. (2005). Revista Contabilidade \& Finanças - USP: uma comparação entre os períodos 1989/2001 e 2001/2004. Revista Contabilidade \& Finanças. São Paulo, 39(3):20-32.

VANTI, N. A. P. (2002). Da bibliometria à webometria: uma exploração conceitual dos mecanismos utilizados para medir o registro da informação e a difusão do conhecimento. Ciência da Informação. Brasília, 31(2):152-162.

Data de Submissão: 26/02/2010

Data de Aceite: 23/03/2010 